\title{
A Mixed-Methods Study Protocol for Soma Experiencing Motion Program (Soma e-motion Program): The Effectiveness of Contemplative Movement for Emotion Regulation
}

\author{
Mi-Sun Lee ${ }^{1}$, Sun Je Kim², Jeong-Ho Chae ${ }^{3}$, E-Jin Park', \\ Wang Yeon Won ${ }^{3}$, Yang-Whan Jeon ${ }^{3}$, and Hyu Jung Huh ${ }^{3 凶}$ \\ ${ }^{1}$ Suicide and School Mental Health Institute, Hallym University College of Medicine, Anyang, Republic of Korea \\ ${ }^{2}$ Soma Yoga Movement Center, Seoul, Republic of Korea \\ ${ }^{3}$ Department of Psychiatry, College of Medicine, The Catholic University of Korea, Seoul, Republic of Korea
}

\begin{abstract}
Somatics refers to body work and movement study that emphasize internal perception and experience. Recently, a new perspective has emerged that views somatics-based techniques as a kind of mindful movement. Somatic techniques as contemplative movement can improve emotional regulation ability through improvement of body awareness or interoception. Based on this background, the present study attempts to develop a somatics based program suitable for a group of clinical patients suffering from emotional dysregulation. This study plans to collect quantitative and qualitative data in order to clarify how interoception and the related emotional regulation ability change after the program. These findings will help to explore whether the somatics technique has potential as an emotion regulation program in the future. In addition, the results are expected to contribute to finding an alternative treatment modality for patients who have not achieved a sufficient effect with conventional psychotherapy.

Psychiatry Investig 2021;18(6):500-504
\end{abstract}

Key Words Somatics, Mindfulness, Emotion regulation.

\section{INTRODUCTION}

\section{Somatics as a contemplative movement intervention}

Somatics refers to body work and movement study that emphasize internal perception and experience. "Soma", a term often used in various forms of motion-based therapy, focuses more on "the body as perceived from within", not "the external observation of body and movement by others". The pioneers of somatics developed various techniques to increase physical awareness based on their experiences while recovering from various difficulties, including physical injury. ${ }^{2}$ In recent years, a new perspective has emerged that views somatics-based techniques as a kind of mindful movement. ${ }^{3}$ From this perspective, somatics techniques were conceptualized as movement-based contemplative practices. ${ }^{4}$

\footnotetext{
Received: February 25, 2021 Accepted: April 11, 2021

$\square$ Correspondence: Hyu Jung Huh, MD, PhD

Department of Psychiatry, Incheon St. Mary's Hospital, The Catholic University of Korea College of Medicine, 56 Dongsu-ro, Bupyeong-gu, Incheon 21431, Republic of Korea

Tel: +82-32-230-5059, Fax: +82-32-230-5059, E-mail: arbor99@gmail.com

(a) This is an Open Access article distributed under the terms of the Creative Commons Attribution Non-Commercial License (https://creativecommons.org/licenses/bync/4.0) which permits unrestricted non-commercial use, distribution, and reproduction in any medium, provided the original work is properly cited.
}

Over the past decades, cognitive neuroscience has shifted from prominently disembodied and computational views to more embodied and situated views of the mind. The embodied views of mind postulate that mental functions cannot be fully understood without reference to the physical body and the environment in which they are experienced. ${ }^{5,6}$ Within the field of contemplative science, directing attention to bodily sensations has been studied in the contexts of seated meditation and mindfulnesspractices. ${ }^{7}$ Furthermore, the cultivation of interoceptive, proprioceptive and kinesthetic awareness lies at the core of many movement-based contemplative practices, such as yoga, qigong, and TaiChi, as well as modern somatic therapeutic techniques, such as the Feldenkrais method and the Alexander technique. These practices are grounded in the concepts of embodiment, movement and contemplation, primarily through the lens of an enactive approach to cognition. ${ }^{4}$

\section{Contemplative movement, interoception and emotion regulation}

Emotions include both cognitive elements and body change, such as palpitation, tremor or tension, behavioral or impul- 
sive elements, changes in attention, and feelings of consciousness. ${ }^{8}$ In particular, somatic theories emphasize that body change is a key element of emotion. ${ }^{9}$ The most important concept in understanding the changes in the body connected with emotion is interoception. ${ }^{10}$ Interoception refers to the sensations occurring in various internal organs in the body, and this concept refers to the process of consciously recognizing various changes in the body by processing various signals in the central nervous system from the internal organs. ${ }^{11}$

The physiological elements of emotion include changes in the internal organs that are affected by the autonomic nervous system, and the change in the sensory response signal reflects not only body homeostasis, but also feedback on emotion-related body changes. ${ }^{12,13}$ Therefore, the degree of information processing ability related to interoception seems to play an important role in emotion regulation, and treatment or intervention to improve interoception will help improve overall emotion regulation ability. Somatic techniques as contemplative movement can improve emotional regulation ability through improvement of body awareness or interoception.

\section{Emotion regulation program based on a somatic intervention: the soma e-motion program}

The Soma e-motion program is a program that integrates several existing somatics techniques, such as Feldenkrais, yoga, and soma movement, so they can be easily be administered to patients with emotion dysregulation. The processes of recognizing the sensations that occur inside the body in a curious non-judgmental manner and adjusting them for stabilization of the body and mind are very important for recovery. ${ }^{14,15}$ Traditional seated meditation can be difficult for patients with severe psychopathology. ${ }^{16}$ Traditional seated meditation requires observation and awareness of rising thoughts and body sensations, but this is not an easy task for patients who regularly suffer from negative thoughts and uncomfortable body sensations. ${ }^{15}$ They sometimes experience overwhelming negative thoughts and sensations during the traditional form of meditation. Somatics-based techniques can help these patients learn mindfulness in alternative ways. As a form of contemplative movement, somatics can enhance body awareness, reduce negative ruminant thinking, and further enhanceself-compassion. ${ }^{17-20}$

Based on this background, the Soma e-motion program has been modified and integrated in several aspects so that the somatics technique can be more easily applied to patients with emotion dysregulation. First, in consideration of psychiatric patients with a very low tolerance to pain or uncomfortable sensations, the program is composed of easier and more comfortable movements. ${ }^{21}$ For example yoga for the purpose of physical fitness may be difficult for patients who cannot withstand uncomfortable sensations or pain. Second, how to guide patients rather than the movement itself may be a more important issue, and the program provides more non-instructive guidance. ${ }^{22}$ We carefully considered how the program could allow patients to make their own choices and decisions based on their own experience throughout treatment. We wanted to guide patients to individually adjust the intensity and speed of their movements without effort and give them permission to decide when to stop the tough movements. It is expected that this process will help patients make decisions and approach their life as an independent subject. Third, the program does not use a hands-on technique, which is commonly used in somatics. ${ }^{23}$ We considered that patients who were not strong enough in their ego or who experienced trauma that had violated their body boundary might be vulnerable to hands-on techniques. ${ }^{24}$ Lastly, when constructing the sequence of movements and postures, we considered patients who could easily be tense in an unfamiliar environment. For example, a movement in a supine position can help facilitate body awareness, but such movements are usually done while sitting or standing, considering that lying in an unfamiliar environment at the beginning of treatment can cause excessive strain to some patients. The principle and direction of the soma-emotion program is described in Table 1 . The program is

Table 1. The principle and direction of soma e-motion program

1. First person viewpoint: The basic direction of the program is to pursue the process of exploring one's body and mind from one person's point of view, not from the point of view of others.

2. Non-instructive: The therapist's non-instructive attitude, language, and guidance help the patient to find a comfortable and stable way of movement.

3. Non-judgemental: There is no criterion for being right or wrong in the course of the patient's attempt to move, and no attempt is made to suggest correct posture or alignment. It aims to help patients move "as easily and smoothly as possible" and aims to recognize the possibility of doing it on their own and the limits of unreasonable movement.

4. Simple and easy movement: By repeating a guide movement with simple intention several times, it is possible to increase the density of inner sense and the power of awareness.

5. Autonomy: Throughout the program, the awareness that the patient can control and choose their movement for himself becomes a therapeutic tool. 
four sessions in total and takes 50-60 minutes per session. Each session consists of body scan, breathing training, and soma movement. Table 2 describes the session configuration in detail.

\section{METHODS}

\section{Study aims}

The study will examine the possible mechanisms through which the soma e-motion program may contribute to the improvement of emotion regulation ability. For this purpose, both quantitative data based on self-report scales as well as qualitative data from subject interviews after the program will be collected.

\section{Participants}

Participants will consist of the following: 1) patients diagnosed with depression/anxiety disorder at a general hospital who are receiving treatment, 2) patients participating in a recovery program at a regional addiction rehabilitation center, or 3) graduate students taking psychology classes at a local university. Among these, the 4th session program will be conducted for patients who have agreed to participate in this study and provided written informed consent.

\section{Study design and procedure}

As there are few research results on how the somatics tech- nique affects emotion regulation, this study design will use a mixed method. This mixed methods study combines qualitative and quantitative methods to strengthen the internal and external validity of the findings. ${ }^{25,26}$ In mixed methods research, the advantages of each method complement one another, and their shortcomings are considerably offset. To collect the quantitative data, a single group pretest-posttest design will be used. The study protocol was approved by the Institutional Review Board of the Ethics Committee of The Catholic Medical Center (KC18FESI031).

\section{Qualitative data collection}

Qualitative data will be acquired from interviews consisting of standardized questions after the fourth session of the program. The questions will be used to gather qualitative information about how participants thought the program helped improve their symptoms and what difficulties they had in participating in the program. The list of standardized questions that will be presented to participants is summarized in Table 3 .

\section{Quantitative data collection}

\section{Interoceptive awareness}

Interoceptive awareness will be measured by the Korean Multidimensional Assessment of Interoceptive Awareness (K-MAIA) ${ }^{27}$ The MAIA is a self-report, 6-point, Likert scale (0: never; 5: always)questionnaire with 32 items within the

Table 2. The main components of soma e-motion program

Body scan
(eg, touch, weight, length, etc.) of the body parts that touch the floor.
Breathing training $\quad$ Rather than instructing the movement and breathing, the patient is guided to detect when and how to breathe and
what movement and breathing to feel tense and relaxed. The breathing guide in this program is different from the
practice of focusing and observing breathing in meditation and yoga, and is a process of finding your own natural
breathing.
In an easy, smooth and slow moving process, patients can learn to recognize the parts of the body that are tense and
relaxed, and select and control the movements they need.
1st session: Exploring the length of the spine and the width of the back of the body based on pelvic movement
2nd session: Exploration physical tension and breathing patterns based on eye movement
3rd session: Exploring the lateral part of the body based on tilting movement
4th session: Exploring tension and relaxation of moving body parts related to respiration based on one's own hands-on

Table 3. The list of standardized questions

1. How has the Soma e-motion program helped you deal with your emotions?

2. Have you ever used the things you learned through the SOMA Movement Program in your daily life? What situations and feelings did you use? Also, what kind of help did you get when you used it?

3. Did you have any difficulties while participating in the Soma e-motion program? Please tell us if there are any areas that you wish to be improved a little more.

4. Was there any movement or posture that was particularly difficult among those you did in the Soma Movement Program? If yes, please tell me. 5. Did you feel that session 4 was appropriate? Or did you think it would be longer or shorter? 
following eight scales that measure interoceptive bodily awareness: 1) Noticing, 2) Not-Distracting, 3) Not-Worrying, 4) Attention Regulation, 5) Emotional Awareness, 6) Self-Regulation, 7) Body Listening, and 8) Trusting.

\section{Rumination}

Ruminative response will be measured by the Korean version of the Rumination Response Scale (K-RRS). ${ }^{28}$ The ruminative response scale includes 22 items describing responses to depressed mood that are self-focused, symptom-focused, and focused on the possible causes and consequences of dysphoric mood. Each item is rated on a Likert scale ranging from 1 (almost never) to 4 (almost always).

\section{Self-compassion}

The Self-compassion Scale (SCS) is a widely used self-report measure developed to assess six components of self-compassion: self-kindness, self-judgment, common humanity, isolation mindfulness and over-identification. ${ }^{29}$ Twenty-six items are rated on a 5-point scale (e.g., $1=$ almost never to $5=$ almost always).

\section{Data analysis}

\section{Qualitative data collection}

A qualitative analysis will be conducted following the guidelines of the grounded theory approach. ${ }^{30,31}$ The data will be coded to identify recurring, similar and contrasting patterns of content. Constant comparison and categorization of codes will be conducted across and within the different focus groups. Similar codes in terms of their characteristics and nature will be aggregated into themes and subthemes. Then, theory development will take place. According to the grounded theory approach, inductive processes are employed to move from the specifics in the data to the more generic and larger-scale concepts supported by the raw data. Finally, a member-checking procedure will be used to validate the findings by the participants. $^{32}$

\section{Quantitative analysis}

Evaluation of within-subject differences between pretest and posttest will be performed using a paired t-test or Wilcoxon signed-rank tests.

\section{Mixed method analysis}

A merged data analysis will be conducted based on a sideby-side comparison to assess for confirmation, expansion or discordance between the datasets, as well as to draw metainferences. ${ }^{25,33,34}$ Confirmation will occur if the findings from both types of data reinforce the results from the other. Expan- sion will occur if the findings from the two datasets diverge and expand insights by addressing different or complementary aspects of the participants' experiences. Discordance will occur if the qualitative and the quantitative results are inconsistent or contradictory. ${ }^{25,33}$ Meta-inferences will include the overall conclusions, interpretations, explanations or knowledge gained from the integration of the two data sets. ${ }^{25}$ This procedure will help assess the extent to which the quantitative data provide for a more complete understanding of participants' experiences and views in an attempt to construct a theoretical model. ${ }^{33,34}$

\section{DISCUSSION}

In recent years, cognitive science has changed from the perspective of seeing the mind as a separate entity from the body to the perspective that various functions of the mind have an inseparable relationship with the body and the surrounding environment. ${ }^{4,635-37}$ Following this shift, various techniques of somatics that deal with body work or movement that emphasize sensory experiences from a first-person perspective are gaining attention. ${ }^{22}$ The techniques of somatics promote awareness of the body's senses, called interoception, through moving the body, and help participants to observe themselves from a distance by connecting them with their own thoughts and emotions. ${ }^{10,12,13}$ In this context, somatics techniques have the potential to be an easier way to learn a mindful attitude for patients with severe psychopathology who have difficulty with traditional forms of meditation. ${ }^{4}$ In order for the program to be useful in clinical practice, it is necessary to consider the different characteristics of patients while taking advantage of the somatics technique. In addition, studies on the clinical effects and mechanisms are needed.

Based on this background, this study attempts to develop a program suitable for a group of clinical patients suffering from emotional dysregulation. This study plans to collect quantitative and qualitative data in order to clarify how interoception and the related emotional regulation ability change after the program. These findings will help to explore whether the somatics technique has potential as an emotion regulation program in the future. In addition, the results are expected to contribute to finding an alternative treatment modality for patients who have not achieved a sufficient effect with conventional psychotherapy. Finally, by demonstrating a scientific basis that specifically clarifies the connection between the body and emotion, this study will contribute greatly to the treatment of patients suffering from emotional dysregulation in the future. 


\section{Acknowledgments}

This research was supported by a Grant of Translational R\&D Project through Institute for Bio-Medical convergence, Incheon St. Mary's Hospital, The Catholic University of Korea.

\section{Conflicts of Interest}

The authors have no potential conflicts of interest to disclose.

\section{Author Contributions}

Conceptualization: all authors. Data curation: Hyu Jung Huh. Formal analysis: Hyu Jung Huh, Mi-Sun Lee. Funding acquisition: Hyu jung Huh. Investigation: all authors. Methodology: Hyu Jung Huh, Mi-Sun Lee. Project administration: Hyu Jung Huh. Resources: Hyu Jung Huh. Software: Hyu Jung Huh. Supervision: Hyu Jung Huh. Validation: Hyu Jung Huh, Jeong-Ho Chae. Visualization: Mi-Sun Lee. Writing_original draft: Mi-Sun Lee. Writing_-review \& editing: Hyu Jung Huh, Mi-Sun Lee.

\section{ORCID iDs}

$\begin{array}{ll}\text { Mi-Sun Lee } & \text { https://orcid.org/0000-0003-2918-0546 } \\ \text { Sun Je Kim } & \text { https://orcid.org/0000-0001-7887-7521 } \\ \text { Jeong-Ho Chae } & \text { https://orcid.org/0000-0002-6070-9324 } \\ \text { E-Jin Park } & \text { https://orcid.org/0000-0002-4352-7131 } \\ \text { Wang Yeon Won } & \text { https://orcid.org/0000-0002-7587-9764 } \\ \text { Yang-Whan Jeon } & \text { https://orcid.org/0000-0002-7516-438X } \\ \text { Hyu Jung Huh } & \text { https://orcid.org/0000-0001-8050-9189 }\end{array}$

\section{REFERENCES}

1. Hanna T. The field of somatics. Somatics 1976;1:30-34.

2. Fortin S. Living in movement: development of somatic practices in different cultures. J Dance Educ 2002;2:128-136.

3. Schmalzl L, Kerr CE. Editorial: Neural mechanisms underlying movement-based embodied contemplative practices. Front Hum Neurosci 2016;10:169.

4. Schmalzl L, Crane-Godreau MA, Payne P. Movement-based embodied contemplative practices: definitions and paradigms. Front Hum Neurosci 2014;8:205.

5. Varela FJ, Thompson E, Rosch E. The Embodied Mind: Cognitive Science and Human Experience. Cambridge, MA: The MIT Press; 1991.

6. Thompson E. Mind in Life: Biology, Phenomenology, and the Sciences of Mind. Cambridge, MA: Belknap Press/Harvard University Press; 2007.

7. Didonna F. Clinical Handbook of Mindfulness. New York: Springer; 2009.

8. Primmer JW. A Somatic-Perceptual Theory of the Emotions. Hamilton, Ontrio: Thesis; 2015.

9. Damasio A. The Feeling of What Happens: Body and Emotion in the Making of Consciousness. Fort Worth, TX: Harcourt College Publishers; 1999.

10. Craig AD. Interoception: the sense of the physiological condition of the body. Curr Opin Neurobiol 2003;13:500-505.

11. Ceunen E, Vlaeyen JWS, Van Diest I. On the origin of interoception. Front Psychol 2016;7:17.

12. Critchley HD, Garfinkel SN. Interoception and emotion. Curr Opin Psychol 2017;17:7-14

13. Craig AD. Interoception and Emotion: A Neuroanatomical Perspective. In: Lewis M, Haviland-Jones JM, Barrett LF, Editors. Handbook of Emotions. 3rd Ed. New York, NY: The Guilford Press, 2008, p. 272-292. 14. Kabat-Zinn J. Full Catastrophe Living, Revised Edition: How to Cope with Stress, Pain and Illness Using Mindfulness Meditation. Hachette: Piatkus Books; 2013.

15. Bishop SR, Lau M, Shapiro S, Carlson L, Anderson ND, Carmody J, et al. Mindfulness: a proposed operational definition. Clin Psychol 2004; 11:230-241.

16. Kim B, Cho SJ, Lee KS, Lee JY, Choe AY, Lee JE, et al. Factors associated with treatment outcomes in mindfulness-based cognitive therapy for panic disorder. Yonsei Med J 2013;54:1454-1462.

17. Wielgosz J, Goldberg SB, Kral TR, Dunne JD, Davidson RJ. Mindfulness meditation and psychopathology. Annu Rev Clin Psychol 2019; 15:285-316.

18. Raab K. Mindfulness, self-compassion, and empathy among health care professionals: a review of the literature. J Health Care Chaplain 2014;20: 95-108.

19. Patel NK, Nivethitha L, Mooventhan A. Effect of a yoga based meditation technique on emotional regulation, self-compassion and mindfulness in college students. Explore (NY) 2018;14:443-447.

20. Snaith N, Schultz T, Proeve M, Rasmussen P. Mindfulness, self-compassion, anxiety and depression measures in South Australian yoga participants: implications for designing a yoga intervention. Complement Ther Clin Pract 2018;32:92-99.

21. Michaelides A, Zis P. Depression, anxiety and acute pain: links and management challenges. Postgrad Med 2019;131:438-444.

22. Hanna T. What is somatics. Somatics: Magazine-Journal of the Bodily Arts and Sciences 1986;5:4-8.

23. Fraleigh S. Moving Consciously: Somatic Transformations through Dance, Yoga, and Touch. Illinois: University of Illinois Press; 2015.

24. Levine PA. Healing trauma. ReadHowYouWant. com; 2008.

25. Creswell JW, Clark VLP. Designing and Conducting Mixed Methods Research. Washington, DC: Sage Publications; 2017.

26. Tashakkori A, Teddlie C. Issues and dilemmas in teaching research methods courses in social and behavioural sciences: US perspective. Int J Soc Res Methodol 2003;6:61-77.

27. Mehling WE, Price C, Daubenmier JJ, Acree M, Bartmess E, Stewart A. The Multidimensional Assessment of Interoceptive Awareness (MAIA). PLoS One 2012;7:e48230.

28. Morrow J, Nolen-Hoeksema S. Effects of responses to depression on the remediation of depressive affect. J Pers Soc Psychol 1990;58:519-527.

29. Neff KD. The development and validation of a scale to measure selfcompassion. Self Identity 2003;2:223-250.

30. Charmaz K. Constructing Grounded Theory: A Practical Guide through Qualitative Analysis. London: Sage; 2006.

31. Glaser BG. Conceptualization: On theory and theorizing using grounded theory. Int J Qual Methods 2002;1:23-38.

32. Lincoln YS, Guba EG, Pilotta J. Naturalistic Inquiry California. Los Angeles, CA: Sage Publications; 1985.

33. Fetters MD, Curry LA, Creswell JW. Achieving integration in mixed methods designs-principles and practices. Health Serv Res 2013;48: 2134-2156.

34. Guetterman TC, Fetters MD, Creswell JW. Integrating quantitative and qualitative results in health science mixed methods research through joint displays. Ann Fam Med 2015;13:554-561.

35. Varela FJ. Neurophenomenology: a methodological remedy for the hard problem. J Conscious Stud 1996;3:330-349.

36. Varela FJ, Thompson E, Rosch E. The Embodied Mind: Cognitive Science and Human Experience. Cambridge, MA: MIT press; 2016.

37. Thompson E. Sensorimotor subjectivity and the enactive approach to experience. Phenomenol Cogn Sci 2005;4:407-427. 